\title{
Spatial and energy distributions of high energy electron bursts caused by precipitations from the inner radiation belt
}

\author{
Aleksandrin S.Yu.* \\ National Research Nuclear University MEPhI (Moscow Engineering Physics Institute) \\ E-mail: syaleksandrin@mephi.ru
}

\section{Galper A.M.}

National Research Nuclear University MEPhI (Moscow Engineering Physics Institute)

E-mail: AMGalper@mephi.ru

Koldashov S.V.

National Research Nuclear University MEPhI (Moscow Engineering Physics Institute)

E-mail: SVKoldashov@mephi.ru

Mikhailov V.V.

National Research Nuclear University MEPhI (Moscow Engineering Physics Institute)

E-mail: SVKoldashov@mephi.ru

\section{Zharaspayev T.R.}

National Research Nuclear University MEPhI (Moscow Engineering Physics Institute)

E-mail: masscry@gmail.com

This work is devoted to the analysis of experimental data of high-energy electron flux in the nearEarth space. The data were obtained during the ARINA and VSPLESK satellite experiments. The ARINA and VSPLESK spectrometers measured the fluxes of electrons and protons with energies of 3-30 MeV and 30-100 MeV respectively. The ARINA was carried out since 2006 till 2016 on board the Resurs-DK1 satellite. The VSPLESK was carried out since 2008 till 2013 on board the International Space Station. The goal of these experiments was to study the physics origin of highenergy charged particle variations and bursts (short term increases of fluxes). Spatial distributions and energy spectra of high-energy electron busts caused by various local geophysical phenomena were analyzed.

35th International Cosmic Ray Conference - ICRC2017

10-20 July, 2017

Bexco, Busan, Korea

\footnotetext{
* Speaker.
} 


\section{Introduction. Local disturbances of the radiation belt and high-energy electron bursts in the near-Earth space}

Nowday the great interest are caused by study of the changes in radiation conditions in the near-Earth space below the radiation belt, appearing as bursts and variations of charged particle fluxes in a wide energy range. High-energy charged particle bursts were first discovered in 1985 in the MARIA experiment performed by the MEPhI on board the Salyut-7 orbital station [1]. Later, extensive experimental and theoretical studies in this field were fulfilled. The interrelation between particle bursts and various solar-magnetospheric and geophysical (seismic, thunderstorm, etc.) processes was established (see [2]-[11] and references therein). It is necessary to mention the most important carried out satellite experiments: MARIA, MARIA-2 [3, 4, 6], and SAMPEX/PET [7, 9] at high energies (5-50 MeV); Demeter (0.3-5 MeV) [10] and POES (0.3-2.5 MeV) [11] at low energies.

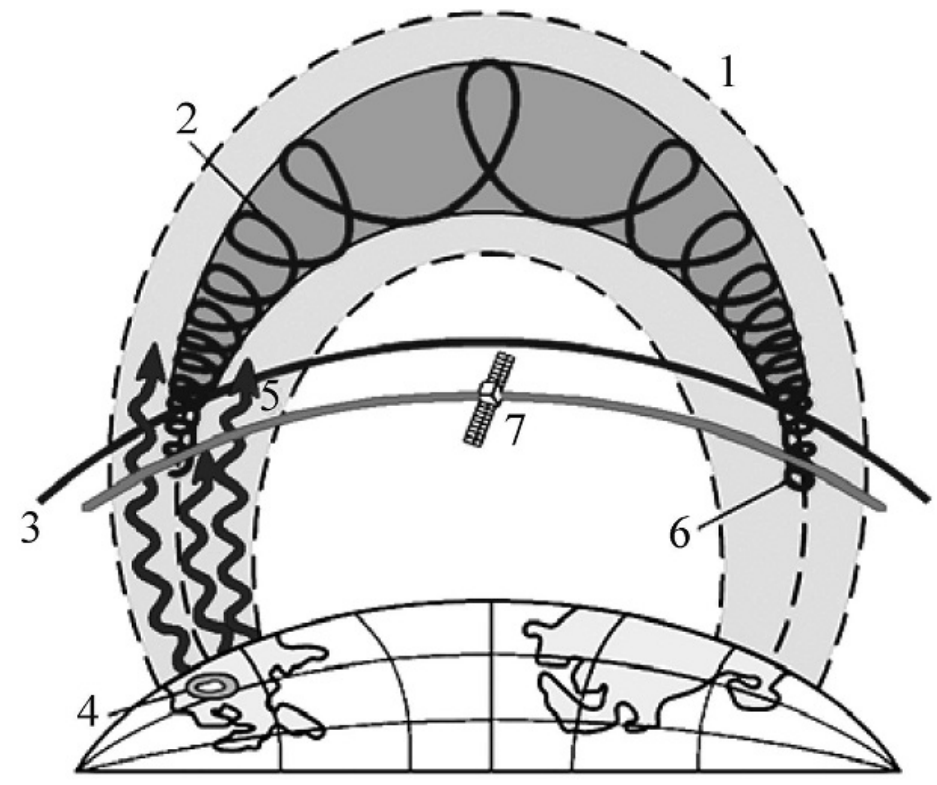

Figure 1: Precipitation of the radiation belt particles after the interaction with EME of seismic origin: (1) geomagnetic tube, (2) particle trajectory, (3) lower boundary of the radiation belt, (4) earthquake epicenter or thunderstorm region, (5) electromagnetic emission, (6) precipitating particles, and (7) satellite trajectory.

These studies showed that the formation mechanism of high-energy charged particle bursts (observations are mostly related to electrons with energies on the order of several tens of $\mathrm{MeV}$ ) is associated with local disturbances of the radiation belt and is as follows [2, 3]. High-energy electrons trapped by geomagnetic field interact with low-frequency electromagnetic emission (EME) generated in various geophysical and magnetospheric processes, which results in pitch-angle diffusion of particles and lowering their mirror points. As a result, electrons precipitate from the radiation belt to altitudes below its boundary. Figure 1 shows this process in the case of seismic or lightning disturbance of the radiation belt. Then precipitated particles, if their mirror points are not too deep in the residual atmosphere (above $60-80 \mathrm{~km}$ ), drift around the Earth and form a wave of precipitated particles (referred to as the GKV wave), propagating along the L-shell containing a 
local disturbance region. The time of the complete longitudinal revolution of high-energy electrons around the Earth is from several tens of seconds to several minutes. Therefore, for same time, the L-shell is completely filled by precipitated particles. When a spacecraft crosses such a perturbed L-shell, instruments register a particle burst which, obviously, can be observed at any longitude not necessarily coinciding with the longitude of the local disturbance region (containing mirror points of electron). As well as electron burst can be formed in the magnetically-coupled zone (containing mirror points of electron), corresponding to this region. Thus, the near-Earth space region, in which particle bursts caused by radiation belt local disturbances can be observed, significantly broadens and the probability of their detection in satellite measurements increases.

In $[3,4,17]$, it was shown that geographical coordinates of the radiation belt local disturbance region, i.e., the position of the region over which particle precipitation occurred (e.g., over the earthquake, thunderstorm activity zone, etc.), can be determined by measuring electron burst characteristics (the detection place, energy spectrum evolution, and time profiles). For particle bursts of seismic origin, it was found that they appear several hours before strong earthquakes. Approaches to the application of this effect for earthquake prognosis are developed [5, 13, 14].

\section{Orbital experiments and instruments}

The ARINA and VSPLESK scintillation spectrometers developed by the MEPhI are fully the sane in physics schemes, have identical physical parameters (geometrical factor, energy range, energy resolution, and others).

The multilayer scintillation detector (ten scintillation layers) is main part of the instruments. Charged particles (electrons, protons) move in the instrument aperture, determining by three first segmented layers, sequentially pass through scintillation layers, lose energy, and are absorbed in the detector. Particles passed through the entire instrument are cut by last bottom layer of detector operating in the anticoincidence mode. Thus, particles stopped in the multilayer detector are electrons with energies of 3-30 MeV and protons with energies of 30-100 MeV. Particles are identified by the energy release in each scintillation layer when they pass through the instrument in combination of particle range in detector layers. The electron and proton energies are measured by their range in detector. The physics scheme and performances of the instruments are described in detail in [15].

Instruments give the possibility to measure the energy spectra of particles with $10-15 \%$ energy resolution and to trace spectra evolution, to determine time profiles of particle flux variations with high time resolution and can operate in high-intensity particle fluxes in the radiation belts or during power solar proton events. The instrument acceptance controlled by the configuration and arrangement of three top detector layers is about $10 \mathrm{~cm}^{2} \mathrm{sr}$. At that instrument field of view is about $20^{\circ}$ and angular resolution within $7-10^{\circ}$. The main objective of experiments is the study of highenergy charged particle bursts of solar-magnetosphere and geophysical origin in the inner zone of magnetosphere.

The ARINA and VSPLESK experiments are carried out on board the low-orbit spacecrafts. The ARINA instrument is installed on board the Resurs-DK1 satellite with an altitude of 350-600 $\mathrm{km}$ and an orbit inclination of $70^{\circ}$, the experiment was executed 2006-2016 [12, 17]. 
The VSPLESK instrument is installed on the International Space Station (altitude is 350-400 $\mathrm{km}$, an orbit inclination is $51^{\circ}$ ). Measurements were carried out in 2008-2013 [13].

In the present study, we used the experimental ARINA and VSPLESK data on the electron flux.

Bursts of high energy charged particles were detected in each experiment. Bursts of electrons at the level of 5 standard deviations and with duration from several seconds to several minutes were selected for further processing and analysis. About 1500 particle bursts were detected in the ARINA and VSPLESK experiments. An example of typical electron burst is presented in Figure 2.

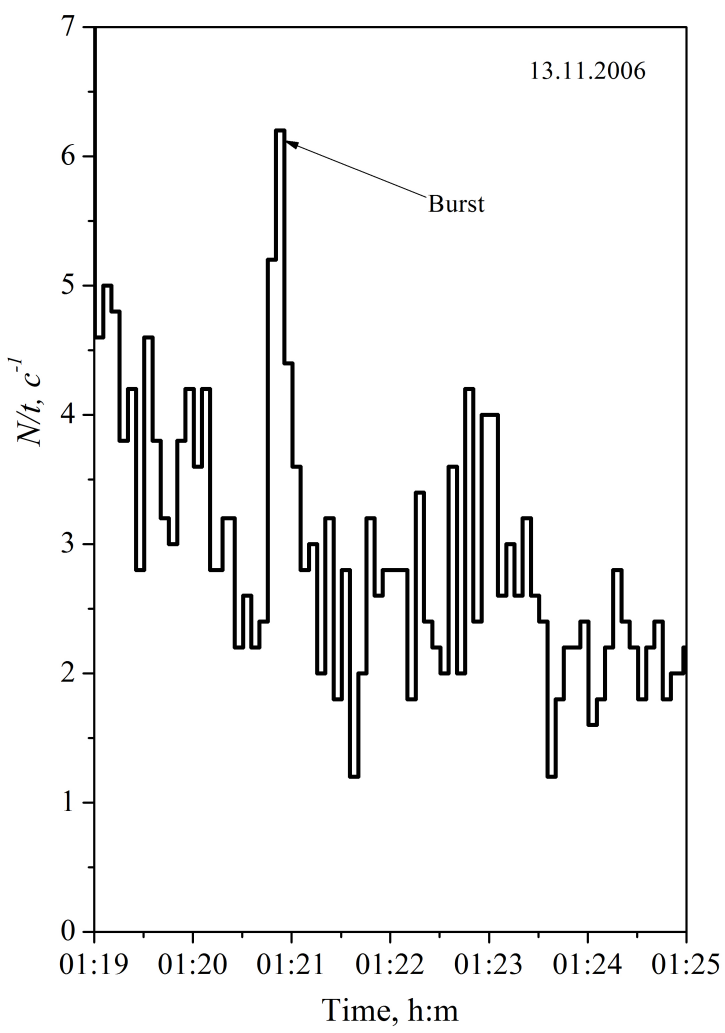

Figure 2: Burst of electrons observed by ARINA spectrometer in space experiment on board the ResursDK1 satellite.

The geographical distribution of particle bursts was studied. L-shells of position of particle bursts detection in the range $1.12-2.0$ were taken in the analysis. That is, it was chosen zone of the near-Earth space where the particles, precipitated from the inner the radiation belt, could be registered. To exclude from the processing the region of the radiation belt the measurements with geomagnetic induction $\mathrm{B}>\mathrm{B}_{o}$ were selected. $\mathrm{B}_{o}$ is the atmospheric boundary of the radiation belt.

\section{Numerical modeling of high-energy electron burst formation in magnetosphere}

After interaction EME with electrons in radiation belt and precipitation of part of electrons as a result of this interaction, a cloud of precipitated electrons moves along drift shell around the 
Earth. Longitudinal drift velocity of particles depends on their energy, L parameter of drift shell and equatorial pitch angle (or particle mirror point latitude).

The time $\left(\mathrm{T}_{d r}\right)$ for particle to perform a complete rotation around the Earth defined by formula 3.1:

$$
T_{d r}\left(E, E_{0}\right)=\frac{88\left(1+E / E_{0}\right)}{2+E / E_{0}} \frac{K}{L E}
$$

where $\mathrm{E}$ is the kinetic energy of the particle, $\mathrm{E}_{0}$ - its rest energy, $\mathrm{L}$ - drift shell (McIlvain coordinate). $\mathrm{K}=1.25-0.25 \cos ^{2}\left(\phi_{m}\right)$, where $\phi_{m}$ is the particle mirror point latitude. $\mathrm{E}, \mathrm{E}_{0}-\mathrm{MeV}, \mathrm{T}_{d r}$ - minutes.

In present work using Monte-Carlo method two sets of electrons were generated and followed all the way to their registration. The first set is electrons precipitated locally and instantly from the radiation belt (cloud of electrons), and the second one consists of background albedo electrons and independent on time. Also it was suggested that energy spectra of trapped and albedo electrons are the same and have a power law shape with index -1.5. This method described in detail in [17].

In drift processing, a cloud of precipitating electrons expands in longitude. So the duration (and size) of this cloud increases. The dependence of cloud duration, containing electrons in 3-30 $\mathrm{MeV}$ range, on longitude distance traveled by electrons is presented in Figure 3.

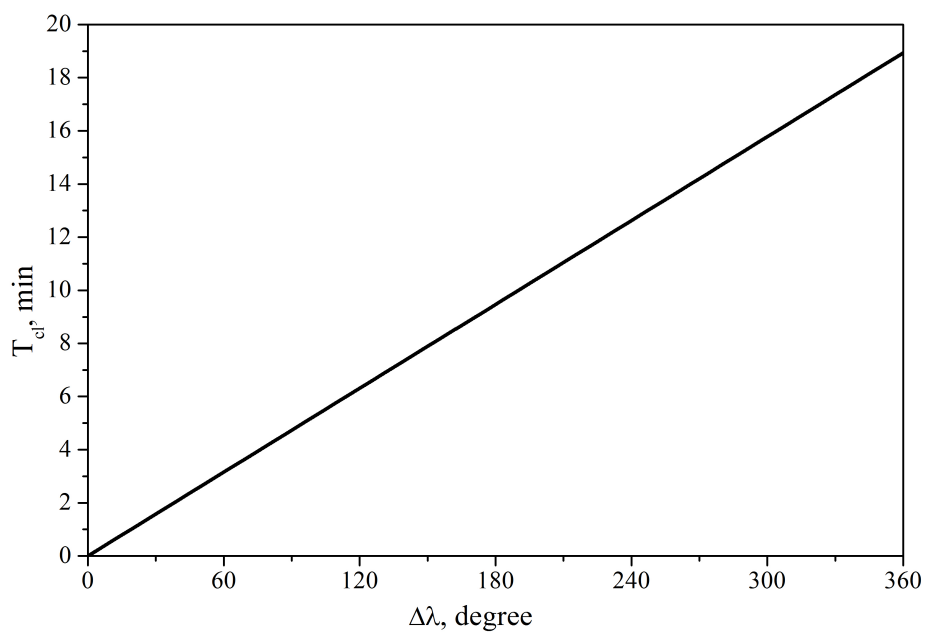

Figure 3: The dependence of cloud duration $\left(\mathrm{T}_{c l}\right)$ on longitude $(\Delta \lambda)$ traveled by drifting electrons (3-30 $\mathrm{MeV})$.

It is important to note that crossing time of $\mathrm{L}$ shell corresponding inner radiation belt $(\mathrm{L}=1-2)$ is about one minute for low orbit satellite with inclination more 40 degree. This time is smaller than cloud duration. In this case there are two different cases of electron burst registration by satellite. The first case is the cross of a head part of drifting electron cloud and the second one is the cross of a tail part of drifting electron cloud. A tail of electron cloud is depleted high-energy part of energy spectrum because of high velocity of longitudinal drift of high-energy electrons. So the tail of electron cloud is enriched with low energy electrons during the drift in difference of a head part of cloud (Figure 4a) 
And for longitudinal distance traveled by electrons of cloud greater than 40-50 degree low energy part of registering electron spectrum at crossing $L$ shell of cloud head by satellite will be depleted. In Figure 4b this effect (depletion of low energy part of electron spectrum) is shown.
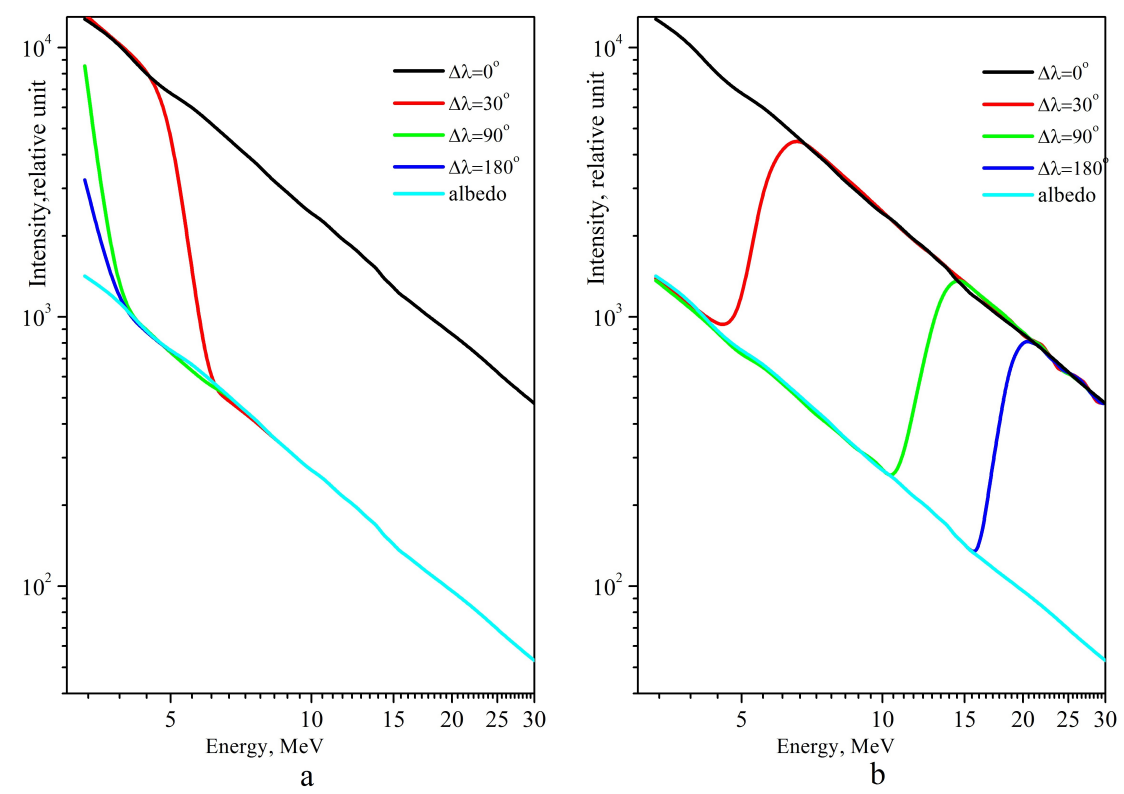

Figure 4: The depletion of high-energy part (a) and low energy part (b) of spectrum of drifting electrons (3-30 MeV).

\section{Comparison modeling and experimental results}

For analysis of possibility using the energy spectra of electron bursts for determination of precipitation regions it was chosen the electron burst with a duration of about one minute, observed by the ARINA instrument on August 24, 2009.

This burst has been used for such purpose earlier in [17]. In this work [17] another method based on energy-time diagram analysis was fulfilled. For new method considered in present paper comparison of energy spectra observed in ARINA experiment and modeled for different conditions $\Delta \lambda$ is presented in Figure 5.

Figure 5 shows that experimental points agree satisfactorily only with the modeled spectrum for $\Delta \lambda=30^{\circ}$. Table 1 presents values of $\chi^{2}$ versus longitude distance traveled by drifting electrons $(\Delta \lambda)$. Region of electron precipitation from the radiation belts determined in this work matches with [17]. It is important to note that approach considered in present paper increases the reliability of the results because does not require utilization of complex mathematical methods under low statistics.

Table 1.

\begin{tabular}{|c|c|c|c|c|}
\hline$\Delta \lambda$ & $0^{\circ}$ & $30^{\circ}$ & $45^{\circ}$ & $90^{\circ}$ \\
\hline$\chi^{2}$ & 10 & 3 & 9 & 13 \\
\hline
\end{tabular}




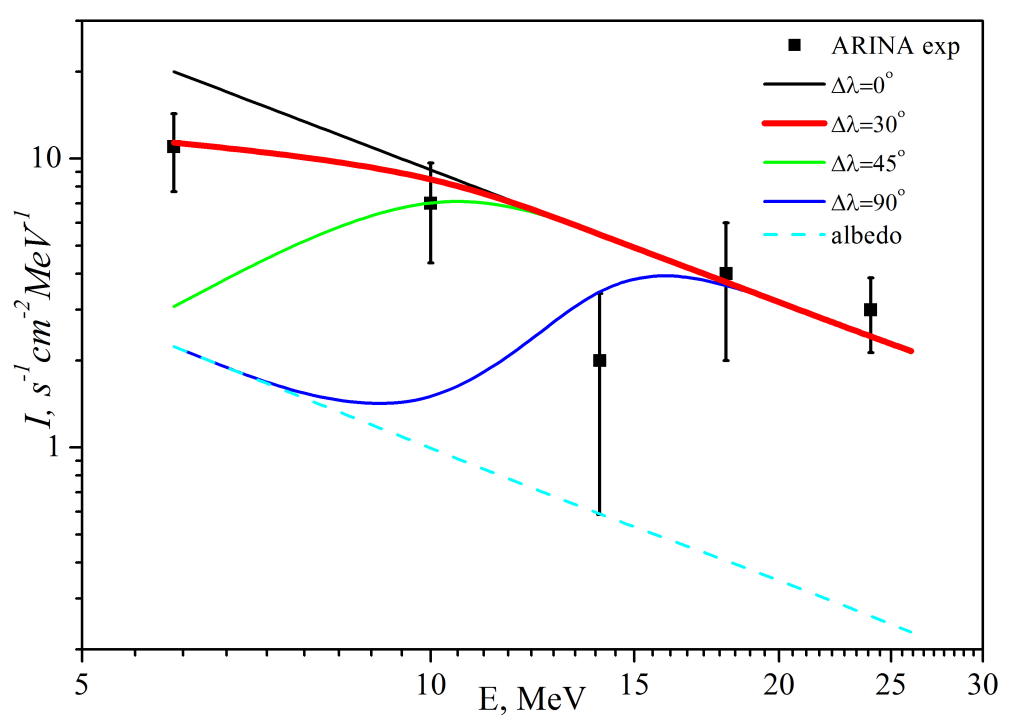

Figure 5: Energy spectrum of the electron burst observed in ARINA experiment 24 August 2009 (square points) and results of modeling for different longitudinal distance traveled by electron cloud.

\section{Conclusion}

Numerical modeling results considered in this work show the changing energy spectrum of bursts of electrons generated by precipitation of electrons under local disturbances of the inner radiation belt with subsequent drift of electrons along L shell around the Earth. Energy spectrum of bursts of drifting electrons depends on the longitudinal distance traveled by drifting cloud of electrons. So it is possible to determine the place of precipitation of particles from the radiation belt with using comparison of energy spectrum of electron burst, observed in satellite experiment, and modeled one. This technique has been used for electron bursts observed on 24 August 2009 and result determined for longitudinal distance matched the previously obtained by another method [17].

\section{Acknowledgments}

This work was supported by the Russian Ministry of Education and Science, project No 3.2131.2017.

\section{References}

[1] Voronov S.A., Galper A.M., Kirillov-Ugriumov V.G. et al. Registration of sporadic increase of high energy particle flux near Brasilia magnetic anomaly region. Proc. 20-th Int. Cos. Ray Conf., 1987, v.4, p. $451-452$

[2] A.M. Galper, S.V. Koldashov, A.M Murashov. Cosmic Research. V. 38, No 1, p. 102 (2000)

[3] M.E. Aleshina, S.A. Voronov, A.M. Galper et al,. Cosmic Research. V 30, p.79 (1992)

[4] A.M. Galper, S.V. Koldashov, S.A. Voronov. Advance Space Research. V. 15, p. 131 (1995) 
[5] S.Yu. Alexandrin, S.A. Voronov, A.M. Galper et al. Izvestiya Academiy Nauk, physics series. Vol 65, No 1, p. 400 (2001)

[6] S.Yu. Alexandrin, S.A. Voronov, A.M. Galper et al. Izvestiya Academiy Nauk, physics series. Vol 67, No 4, p. 79 (2003)

[7] S.Yu. Aleksandrin, A.M. Galper, S.V. Koldashov, et al. Annales Geophysicae. V. 21, p. 597 (2003)

[8] S.Yu. Aleksandrin, A.M. Galper, S.V. Koldashov, A.M. Murashov. Izvestiya Academiy Nauk, physics series. Vol 69, No 6, p. 872 (2005)

[9] V. Sgrigna, L. Carota, L. Conti, et al. J. Atm. Solar-Terrestrial Phys. V. 67, p. 1448 (2005)

[10] M. Parrot, J.J. Berthelier, J.P. Lebreton, et al. Physics and Chemistry of the Earth. V. 31, p. 486 (2006)

[11] C. Fidani, R. Battiston, W.J. Burger. Remote Sensing. V. 2, p. 2170 (2010)

[12] A.V. Bakaldin, A.G. Batischev, A.M. Galper et al. Cosmic Research. Vol 45. No 5. p. 471 (2007)

[13] S.Yu. Alexandrin, A.V. Bakaldin, A.G. Batischev, A.M. Galper et al. Izvestiya Academiy Nauk, physics series. Vol 73, No 3, p. 379 (2009)

[14] S.Yu. Alexandrin, A.M. Galper, S.V. Koldashov, et al. Proceedings of the 31-th Russian Conference on Cosmic Rays, Moscow, MSU, 2010, GEO9

[15] A.G. Batischev, S.A. Voronov, S.V. Koldashov et al. Pribory i Tekhnika Eksperimenta. No 5, p. 25 (1999)

[16] A.M. Galper, S.V. Koldashov, A.A. Ulitin. Kratkie soobscheniya po fizike. 2011, No 7, p.41

[17] S. Yu. Aleksandrin, A. M. Galper, T. R. Zharaspayev, and S. V. Koldashov Bulletin of the Russian Academy of Sciences: Physics, 2015 Vol. 79, No. 5, pp. 646-648. 